\title{
Localized Survival of Ciliary Ganglionic Neurons Identifies Neuronotrophic Factor Bands on Nitrocellulose Blots ${ }^{1}$
}

\author{
THOMAS B. CARNOW, ${ }^{2}$ MARSTON MANTHORPE, GEORGE E. DAVIS, AND SILVIO VARON
}

Department of Biology and School of Medicine, University of California, San Diego, La Jolla, California 92093

\begin{abstract}
A novel and sensitive method has been developed to identify ciliary neuronotrophic factors (CNTFs) from tissue extracts after blotting to nitrocellulose paper. The CNTF proteins are required for the in vitro survival of embryonic chick ciliary ganglionic neurons. Tissue extracts containing such CNTFs are electrophoresed using sodium dodecyl sulfate-polyacrylamide gel electrophoresis and transferred to nitrocellulose paper. Purified ciliary ganglionic neurons are seeded on the surface of the nitrocellulose blot, and the culture is incubated for $24 \mathrm{hr}$ in medium lacking CNTF. CNTF can be localized on the blot because it retains its ability to support the survival of the neurons cultured on the nitrocellulose. $A$ band of viable neurons, easily visualized by staining with a vital dye, is supported by the blotted CNTF polypeptide. The number of neurons surviving on the blotted CNTF is related to the amount of CNTF originally loaded on the electrophoretic gel. As little as 2 ng (16 trophic units) of CNTF protein contained in crude tissue extracts can be loaded on the sodium dodecyl sulfate gel and still be recognized by the cultured neurons. This method was used to identify CNTF polypeptides from extracts of adult rat nerve $(24,000$ and 19,000 daltons) and from tissue found near experimentally induced adult rat brain lesions (24,000 daltons). The electrophoretic mobilities of these peptides are distinct from the previously purified chick eye CNTF polypeptide $(20,400$ daltons).
\end{abstract}

Neuronotrophic factors may be required by different types of neurons, from the peripheral and central nervous systems, for survival and functional maintenance in vivo and in vitro (for reviews see Varon and Adler, 1981; Varon et al., 1983b, 1984; Manthorpe and Varon, 1985). Nerve growth factor (NGF), the best characterized neuronotrophic factor, supports the survival of embryonic sensory and sympathetic neurons (e.g., Greene and Shooter, 1980). The composition of 75 NGF from mouse submaxillary glands and the amino acid sequence of its biologically active subunit, $\beta$-NGF, are known (for review see Bradshaw and Costrini, 1983). A second neuronotrophic factor, distinct from NGF, was isolated from pig brain

Received August 13, 1984; Revised January 11, 1985;

Accepted January 16, 1985

1 This work was supported by United States Public Health Service Grant NS 16349. T. B. C. was supported by National Institute of Neurological and Communicative Disorders and Stroke Postdoctoral Fellowship 1F32 NS06503-01A1.

${ }^{2}$ To whom correspondence should be sent, at his present address: Department of Biochemistry, University of Connecticut Health Center, Farmington, CT 06032 .
(Barde et al., 1982). It has been reported to have an $M_{r}$ of about 12,300 , and to support the survival of embryonic day $(E) 10$ to $E 15$ chick dorsal root but not of sympathetic or ciliary ganglionic neurons.

A ciliary neuronotrophic factor (CNTF) has recently been purified from selected chick eye tissues on the basis of its being required for the in vitro survival of neurons from E8 chick ciliary ganglia (Barbin et al., 1984; Manthorpe and Varon, 1984). The purified chick CNTF also displays trophic activity for avian and rodent sensory and sympathetic ganglionic neurons. It has an apparent $M_{r}$ of 20,400 and is distinct from NGF and the pig brain factor with regard to both its target neurons and its molecular properties. CNTF activity is present in high amounts in adult rat sciatic nerve extracts (Williams et al., 1984) and also has been found to accumulate locally in neonatal and adult rat brain in response to experimentally induced lesions (Manthorpe et al., 1983b; Nieto-Sampedro et al., 1983).

Trophic titers of various factors are determined using in vitro bioassays (for review see Varon et al., 1983a). The neuronal target cells are incubated with serial dilutions of the trophic factor source, and the number of neurons surviving at selected culture times reflects the concentration of the trophic factor in the test culture. We describe here a new method to recognize polypoptides in tissuc extracts that have trophic activity for ciliary ganglionic neurons. The polypeptides of the crude material are separated by sodium dodecyl sulfate (SDS-PAGE) and electrophoretically transferred to nitrocellulose. The resulting blots are then incubated with purified neurons from E8 chick ciliary ganglia to probe for CNTF activities. After 24 hr of incubation, living neurons are identified using a vital stain. Only those neurons supplied with CNTF will survive on these nitrocellulose blots and, thus, the CNTF-supported, dye-stained neurons define a band coincidental with the electrophoretic position of the CNTF polypeptide. This demonstrates that CNTFs retain their trophic activity throughout electrophoresis and subsequent binding to nitrocellulose. Using this technique, the electrophoretic behaviors of CNTFs from chick eye and rat nerve extracts and from rat brain lesion fluid are compared.

\section{Materials and Methods}

SDS-PAGE and transfers of protoins to nitrocollulose. Polypeptides werc separated by SDS-PAGE according to the method of Laemmli and Favre (1970) using a 7.5 to $20 \%$ polyacrylamide gradient, and then were transferred electrophoretically to nilrocellulose paper (Millipore, $0.45-\mu \mathrm{rl}$ pore size) dccording to the method of Towbin et al. (1979). Transfer was performed at $500 \mathrm{~mA}$ for $2 \mathrm{hr}, 150 \mathrm{~mA}$ for 12 to $18 \mathrm{hr}$, and finally $600 \mathrm{~mA}$ for $1 \mathrm{hr}$ without cooling. I he distance between electrodes was $3 \mathrm{~cm}$.

Incubation of blots with purified ciliary ganglionic neurons. After electrophoretic transfer of the proteins, the nitrocellulose sheet was rinsed with phosphate-buffered saline (PBS) and cut into strips $(0.5 \times 10 \mathrm{~cm})$ containing individual electrophoretic lanes. Unoccupied protein-binding sites were blocked with $1 \%$ ovalbumin-PBS, $\mathrm{pH} 7.2$, for $2 \mathrm{hr}$ at room temperature in 15$\mathrm{ml}$ capped plastic tubes. Each blot was placed in a shallow rectangular trough $(14 \times 1 \times 1 \mathrm{~cm})$ cut into a block of plastic. The nitrocellulose strips were immobilized flat on the bottom of their trough, and blotted surface facing up, with rings of plastic tubing (outer diameter, $1 \mathrm{~cm}$ ) that were 
wedged against the sides and bottom of the trough at each end of the nitrocellulose strip. The troughs and tubing were sterilized before use by brief treatment with $70 \%$ ethanol and UV irradiation. Once in place, the blots were washed for 15 to 20 min with culture medium (Dulbecco's modified Eagle's medium supplemented to $26.4 \mathrm{mM} \mathrm{NaHCO}_{3}, 2 \mathrm{~mm}$ glutamine, $10 \%$ fetal calf serum, and 100 units $/ \mathrm{ml}$ of penicillin, $100 \mu \mathrm{g} / \mathrm{ml}$ of streptomycin, $0.25 \mu \mathrm{g} / \mathrm{ml}$ of Fungizone).

Ciliary ganglia from E8 chick embryos were dissociated into a single cell suspension in culture medium as described (Varon et al., 1979), and its neurons were enriched (to better than $90 \%$ purity) by differential cell attachment for $2 \mathrm{hr}$ at $37^{\circ} \mathrm{C}$ on tissue culture plastic (Davis et al., 1985). Aliquots of the neuronal cell suspension $\left(2 \times 10^{5}\right.$ neurons in $3 \mathrm{ml}$ of culture medium) were evenly distributed with a Pasteur pipette over each nitrocellulose strip. The troughs were covered with a sterile plastic lid, placed on a level tray, and incubated at $37^{\circ} \mathrm{C}$ in humidified $95 \%$ air $/ 5 \% \mathrm{CO}_{2}$ for 3 or $24 \mathrm{hr}$. In some experiments wider blots containing multiple electrophoretic lanes were placed in larger troughs $(14 \times 4 \times 0.8 \mathrm{~cm})$ and seeded with $5 \times 10^{5}$ neurons in 15 $\mathrm{ml}$ of medium. The surviving neurons were visualized by their ability to form a blue formazan reaction product from a yellow substrate, 13-(4,5-dimethyltheozol-2-yl)-2,5-diphenyl tetrazolium bromide (MTT; Sigma Chemical Co. M2128), as described by Mosmann (1983). After $22 \mathrm{hr}$ of culture, a solution of MTT.PBS $(5 \mathrm{mg} / \mathrm{ml})$ was added to the medium in a volume ratio of $1: 10$ and gently mixed. The neurons were incubated for another 2 to $3 \mathrm{hr}$ and then fixed on the nitrocellulose strips by first adding an equal volume of $2 \%$ glutaraldehyde in Eagle's basal medium. After $15 \mathrm{~min}$, the fixative was replaced with fresh glutaraldehyde medium and the fixation was continued for an additional $30 \mathrm{~min}$. The fixed cultures on nitroccllulose could be stored in PBS at $4^{\circ} \mathrm{C}$ for at least a week. The number of blue-labeled neurons was determined visually within $1-\mathrm{mm}^{2}$ areas by direct counts using light microscopy.

Preparation of tissue extracts. E15 chick intraocular tissues including the choroid, iris-ciliary body, and pigment epithelium (CIPE) were dissected and extracted with water as described previously (Manthorpe et al., 1980). Chick CNTF was purified from CIPE extract according to the method of Barbin et al. (1984) and had a specific activity of $7.7 \times 10^{6}$ trophic units (TU)/mg of protein. Adult rat sciatic nerve extracts, prepared as described by Williams et al. (1984), were concentrated 10-fold using Amicon PM10 ultrafiltration. Gelfoam sponges placed in adult rat entorhinal lesions were collected at 16 days post-lesion and extracted as described by Nieto-Sampedro et al. (1983).

\section{Results}

Survival of ciliary ganglionic neurons on nitrocellulose paper. The in vitro behavior of E8 chick ciliary ganglionic neurons seeded on ovalbumin-blocked, unblotted nitrocellulose strips is illustrated in Figure 1. Neuronal attachment was allowed to proceed for $3 \mathrm{hr}$, and then the MTT was added. After dye exposure, the neurons appeared as round blue cells which were evenly distributed on the nitrocellulose, regardless of the presence of purified chick eye CNTF in the culture medium (Fig. 1A). Neuronal cell density and morphologies appeared unchanged after $24 \mathrm{hr}$ in cultures containing CNTF at 100 $\mathrm{TU} / \mathrm{ml}$ (Fig. 1B). In sharp contrast, very few neurons were stained with MTT after $24 \mathrm{hr}$ in cultures from which CNTF had been omitted (Fig. 1C). Two representative fields of CNTF-supported and CNTF. deprived cultures are shown at higher magnification in Figure 1, $D$ and $E$, respectively. Note the round cell appearance of the dyepositive, viable neurons. The same neurons, if cultured on laminintreated polyornithine substrate (Manthorpe et al., 1983a), would display neuritic outgrowth and, in fact, will even do so in CNTF. supported cultures on nitrocellulose which has been pretreated with laminin (data not shown). The ability of these cells to form neurites, as well as the preponderance $(>90 \%)$ of neurons in the seeded cell population, allowed us to conclude that (i) E8 ciliary ganglionic neurons may be cultured on nitrocellulose substrata and (ii) their survival in this culture system, as in all previously examined culture systems, continues to depend on the availability of CNTF.

Trophic competence of CNTF supplied in a substratum-bound form. We wished to determine whether nitrocellulose-bound chick eye CNTF would retain its ability to provide trophic support to target neurons. Nitrocellulose strips were soaked for $1 \mathrm{hr}$ in $3 \mathrm{ml}$ of culture medium containing purified CNTF $(100 \mathrm{TU} / \mathrm{ml})$ and were rinsed with three changes of PBS for 2 to $3 \mathrm{hr}$; the remaining protein sites were blocked with ovalbumin. The strips were then placed in their troughs and supplied with test neurons in CNTF-free medium. After a 24-hr incubation, the cultures were treated with the dye and examined. Figure $2 \mathrm{~A}$ demonstrates the substantial presence of dye-positive, viable neurons in these cultures. Neuronal morphologies in these cultures appear similar to those in cultures containing CNTF in the media (cf. Fig. 1B).

To examine whether CNTF might be acting locally after binding to the substratum, a small volume $(2 \mu \mathrm{l})$ of CNTF at $10^{6} \mathrm{TU} / \mathrm{ml}$ was "spotted" onto nitrocellulose membranes, the membranes were blocked with ovalbumin, and ciliary neurons were seeded over the entire strip and cultured for $24 \mathrm{hr}$ in CNTF-free medium. Figure 2, B and $C$, shows, respectively, a CNTF-spotted nitrocellulose area after staining with amido black (but without cell seeding) and tested with the vital dye after neuronal culture. Only the CNTF-spotted area contained dye-positive neurons (higher magnification photographs of a spotted and an unspotted area are shown in Fig. 2, D and E),
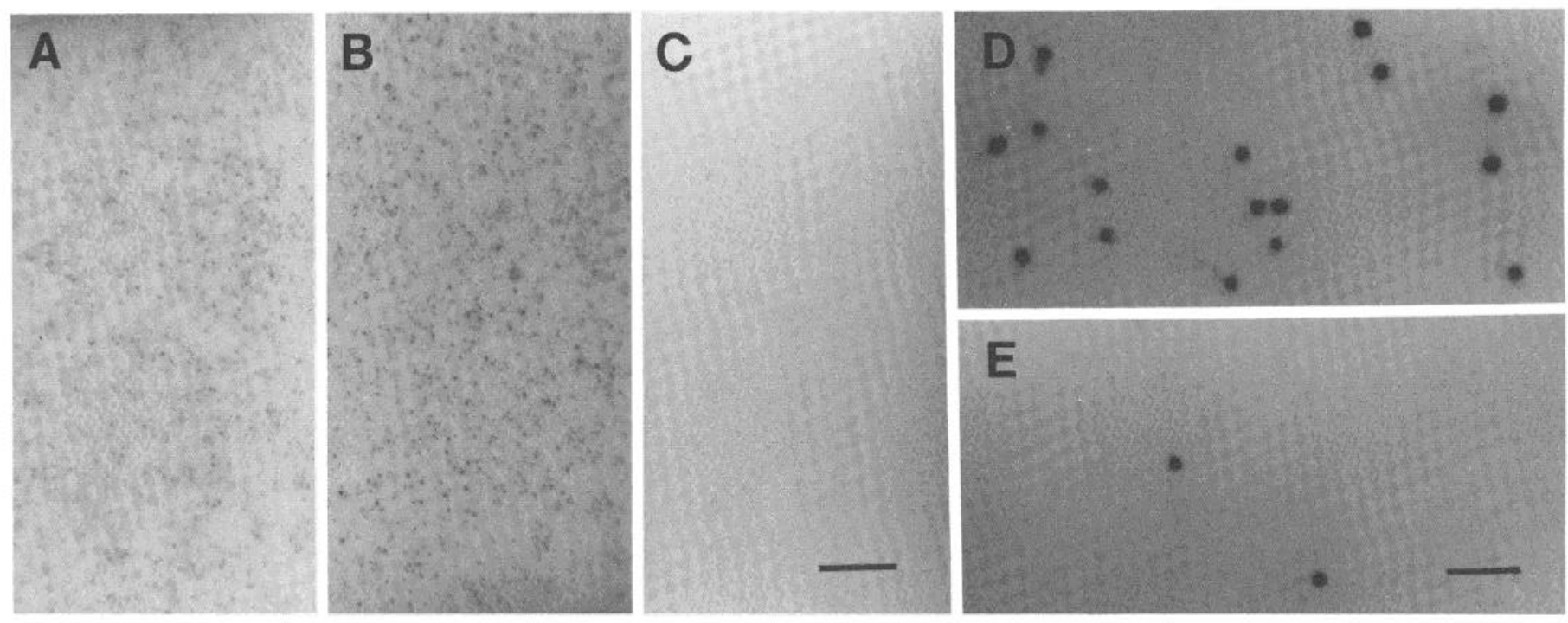

Figure 1. Recognition of dissociated neurons cultured on nitrocellulose paper in the presence or absence of CNTF in the culture medium. Purified ciliary ganglionic neurons were cultured on nitrocellulose paper, stained using a vital dye, fixed with glutaraldehyde, and photographed using light microscopy as described under "Materials and Methods." Typical cultures are shown $3 \mathrm{hr}$ after seeding with no CNTF added $(A), 24 \mathrm{hr}$ with $100 \mathrm{TU} / \mathrm{ml}$ of purified chick eye CNTF in the medium $(B)$, and $24 \mathrm{hr}$ with no CNTF added $(C)$. Higher magnifications of the cultures in $B$ and $C$ are shown in $D$ and $E$, respectively. Note the obvious decrease in the number of stained viable neurons in $C$ and $E$. Magnification bars represent $1.0 \mathrm{~mm}$ in $A$ to $C$ and $100 \mu \mathrm{m}$ in $D$ and $E$. 

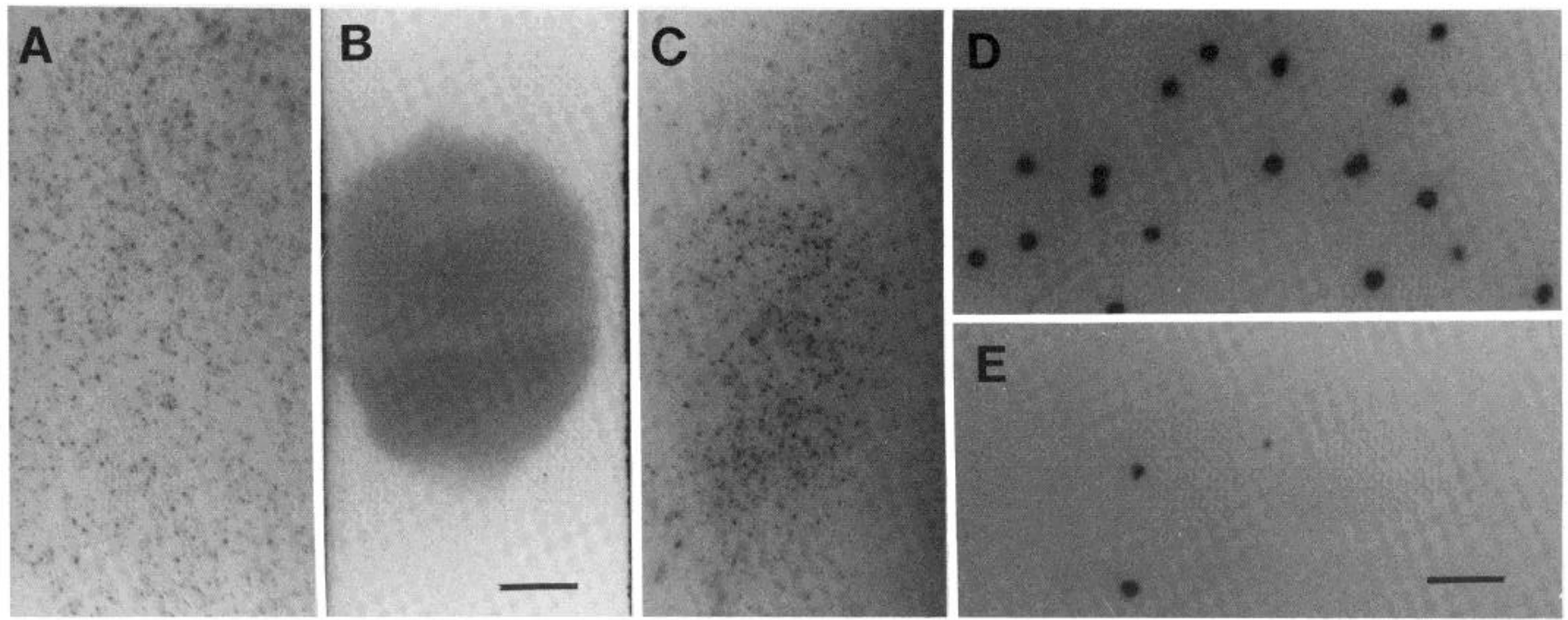

Figure 2. Survival of dissociated neurons on nitrocellulose paper containing bound purified chick eye CNTF. Purified ciliary neurons were cultured for 24 $\mathrm{hr}$ in the absence of CNTF in the medium on strips of nitrocellulose which had been previously treated with purified chick eye CNTF. $A$, Ciliary cultures (24 $\mathrm{hr}$ ) on strips of nitrocellulose which had been uniformly treated with CNTF. B. Amido black stain of a piece of nitrocellulose "spotted" with $2 \mu \mathrm{l}$ of purified CNTF $\left(130 \mu \mathrm{g} / \mathrm{ml}\right.$ of protein and $\left.10^{6} \mathrm{TU} / \mathrm{ml}\right)$. C, Ciliary culture $(24 \mathrm{hr})$ on a nitrocellulose strip (also spotted with $2 \mu \mathrm{l}$ of purified CNTF). Note the localized neuronal survival only on the area containing bound CNTF. Higher magnifications of the culture in $C$ are shown in $D$, an area within the spot, and in $E$, an area outside the spot. Ciliary neurons cultured on CNTF-spotted paper for $3 \mathrm{hr}$ in either the presence or absence of CNTF in the medium, or for $24 \mathrm{hr}$ in the presence of CNTF, appeared indentical to the culture shown in A. Magnification bars represent $1.0 \mathrm{~mm}$ in $A$ to $C$ and $100 \mu \mathrm{m}$ in $D$ and $E$.

indicating that survival of ciliary ganglionic neurons was restricted to the area where CNTF was previously spotted. The dye-positive neurons appeared similarly distributed on both spotted and unspotted areas (i.e., the cultures appeared the same as shown in Figs. $1 B$ and $2 A$ ) at either $3 \mathrm{hr}$ with or without CNTF in the medium or at $24 \mathrm{hr}$ with CNTF included in the medium (data not shown).

The number of dye-positive neurons per unit area was determined using light microscopy, and the results are shown in Figure 3. Neuronal density values are given for $(i)$ a 3-hr culture on CNTF-free nitrocellulose and in a CNTF-free medium, illustrating the neuronal cell density shortly after attachment; (ii) a 24-hr culture on CNTFfree nitrocellulose in the presence of CNTF-supplemented medium (showing the distribution of viable neurons over the entire strip); and (iii) and (iv) a 24-hr culture in CNTF-free medium but with a CNTFspotted nitrocellulose, where neuronal counts were taken within or outside the CNTF-spotted area, respectively. Such measurements clearly indicate that the neuronal cell attachment was independent of either free or bound CNTF. CNTF-dependent neuronal survival was equally promoted by supplying soluble CNTF in the medium or bound CNTF on the nitrocellulose. In the latter case, neuronal survival was precisely restricted to the bound-CNTF area.

CNTF within a crude extract can be recognized after SDS-PAGE and transfer to nitrocellulose. The principle of localized neuronal survival on immobilized CNTF was applied to the detection of CNTF in tissue extracts. Different amounts of chick ocular (CIPE) extract were submitted to SDS-PAGE and blotted to nitrocellulose. The blots were then probed with ciliary ganglionic neurons in CNTF-free medium. The results, shown in Figure 4 , indicate that there was a well defined band, migrating at about 20,000 daltons, which supported the survival of ciliary ganglionic neurons. This apparent molecular weight corresponds to the reported $M_{r}=20,400$ of purified chick CNTF (Barbin et al., 1984). Indeed, purified chick CNTF subjected to SDS-PAGE and blotting occupies a band (revealed by a localized area of surviving neurons) which co-migrates with the 20,000 dalton band from CIPE extracts (data not shown). Neither the purified eye CNTF nor the CIPE extract revealed other CNTF bands with trophic activity for ciliary neurons (cf. Fig. 4B). The intensity and width of the blot band defined by dye-positive cells (Fig. $4 A$ and inset to Fig. $4 B$ ) decreased when less extract was loaded on the gels to be blotted. It is possible to scan the blots along their length using light microscopy, readily identify the regions where "blue" cell density exceeds background, and count dyepositive neurons across the nitrocellulose strips over the positive band region. The greatest neuronal density within the dye-positive area defines a peak representing the electrophoretic mobility of the chick eye CNTF polypeptide. The peak amplitude and area decrease as one decreases CIPE extract loads on the original SDS gel. The study indicates that loads of $10 \mathrm{TU}(<2 \mathrm{ng}$ of protein) of CNTF per lane, whether in the crude CIPE extract or in the purified form, can be detected by this method.

Comparison of CNTFs in different crude sources. Figure 5 compares cultures on blots obtained after SDS-PAGE of two other CNTF sources besides the chick eye. Extracts of adult rat sciatic nerve are particularly rich in CNTF activity (Williams et al., 1984). Mechanical lesions in the cerebral cortex of young or adult rats lead to an accumulation of CNTF activity in both the wound area and the injured brain tissue (Manthorpe et al., 1983b; Nieto-Sampedro et al., 1983). The molecular nature of either of these rat CNTFs has not yet been defined. Use of the present SDS-PAGE and blot approach has revealed, from both rat sources, the occurrence of a 24,000 dalton CNTF protein distinct in electrophoretic mobility from the chick eye CNTF. In one experiment, the rat nerve extract also displayed a second active CNTF band in the 19,000 dalton range distinct from both the 24,000 and 20,000 dalton bands described above. Note, however, that the amount of sciatic nerve extract loaded in this experiment (containing 7000 TU) was relatively high. Indeed, in two other runs using independently prepared extracts, lower activity loads (300 TU) demonstrated only the 24,000 dalton CNTF band (data not shown). The relationship of the CNTF polypeptides from rat sciatic nerve extracts and rat brain lesion fluids to chick eye CNTF remains to be determined. Nevertheless, these findings illustrate that the present blot and culture method can be used to recognize distinct CNTF species within the same or different sample materials.

\section{Discussion}

In the present report we have used SDS-PAGE and nitrocellulose blotting to identify and characterize proteins which support ciliary ganglionic neuronal survival. Our methodological approach was based on that used by Hayman et al. (1982), who established the 


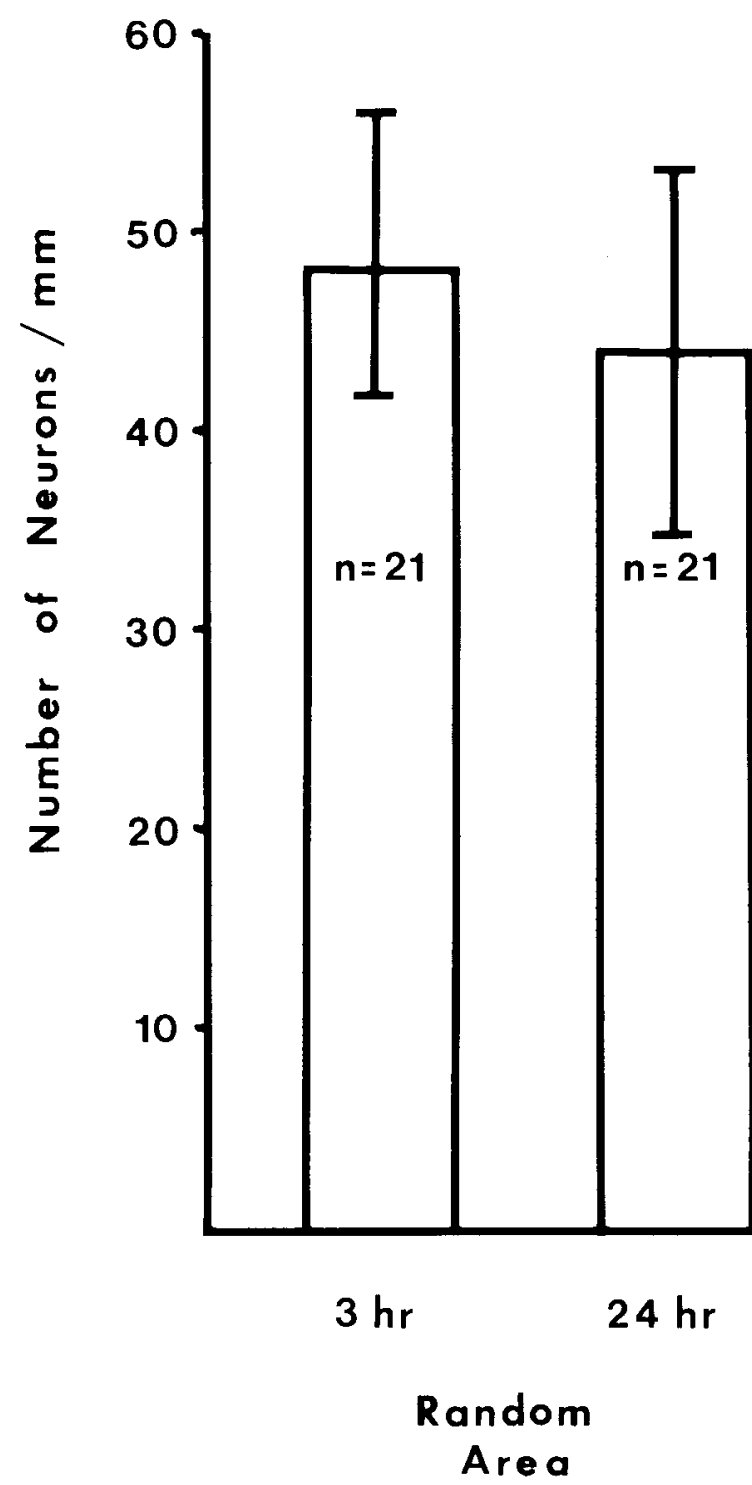

CNTF in Medium

\section{On CNTF Away from Spot}

\section{No CNTF in Medium}

Figure 3. Quantitation of neuronal survival on nitrocellulose paper containing bound purified chick eye CNTF. Ciliary neurons, cultured for 24 hr on CNTFspotted papers similar to those shown in Figure 2, were counted within sampling areas using light microscopy, and the number of ncurons per square millimeter area was determined. Neuronal densities are shown for (1) 3-hr cultures without added CNTF, (2) 24-hr cultures on CNTF-spotted nitrocellulose with CNTF in the culture medium, (3) 24-hr cultures on CNTF-spotted nitrocellulose but without CNTF in the medium, and (4) 24-hr cultures on areas away from the CNTF spot (without CNTF in the culture medium). Values are given \pm SD ( $n=$ the number of individual areas courited).

principle that SDS-PAGE blots of cell attachment-promoting proteins could be probed with normal rat kidney cells to identify biologically active individual polypeptides from plasma.

Methodological prerequisites. The present study uscd E8 chick ciliary ganglionic neurons as probe cells to detect chick and rat CNTFs on nitrocellulose. Three prerequisites were satisfied which permitted the blot system to be successful. First was the demonstration that these neurons attach to nitrocellulose substrata, and that they will survive there only when supplied with a trophic factor in the culture medium. It is reasonable to expect that other probe neurons will behave similarly if provided with an appropriate neuronotrophic agent. Second was the ability of the trophic factor to retain its activity after exposure to SDS and $\beta$-mercaptoethanol during the electrophoretic steps. Purified chick eye CNTF had been shown previously to survive such treatments (Barbin et al., 1984), but there is no assurance that all trophic factors will share this stability. It will be interesting in this regard to adapt the current approach to blots obtained from SDS-free gels, e.g., native PAGE or isoelectric focusing PAGE. The third prerequisite for success in the present method was the capability of a trophic factor to express its activity in culture after having been bound to nitrocellulose.

Applications of the present methodology. The present blot and culture technique, particularly if applied to non-denaturing gel electrophoretic systems, may offer unique experimental approaches to several important questions concerning ciliary ganglionic neuronotrophic factors. A large number of differenl source malerials display CNTF activity (cf. Manthorpe and Varon, 1984) and, as illustrated in Figure 5, the present technique can be used to compare the electrophoretic behaviors of different CNTFs. The technique is particularly well suited for examining CNTFs in samples containing widely different protein and activity contents. This technique should be useful for the detection of apparent molecular weight differences among CNTFs taken from the same tissue at different developmental ages or after experimental treatments of the source cells. Con- 


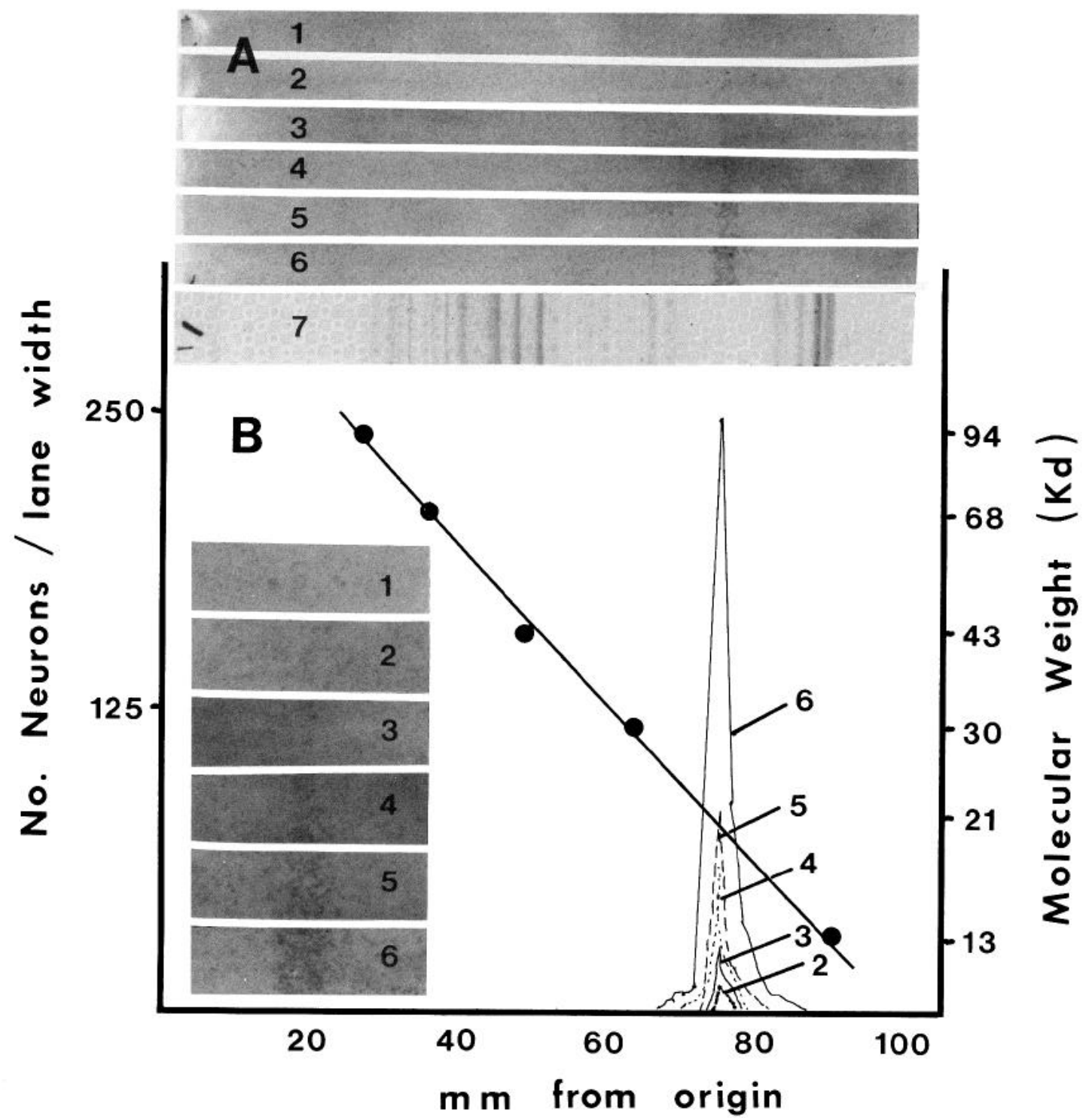

Figure 4. Recognition of nitrocellulose-bound CNTF within an aqueous extract of chick embryo eye. Adjacent individual lanes containing different amounts of CIPE extract were transferred to nitrocellulose after SDS-PAGE, and the resulting blots were probed for CNTF activity using purified ciliary ganglionic neurons as described under "Materials and Methods." A, Photograph of the fixed blot after culture showing ciliary neuronal survival localized only to one discrete band. Each individual lane was loaded with CIPE extract $(400 \mu \mathrm{g} / \mathrm{ml}$ of total protein and $8000 \mathrm{TU} / \mathrm{ml}$ of CNTF activity) containing different amounts of CNTF: lane 1, 7 TU (1 ng); lane 2, 15 TU (2 ng); lane 3, 30 TU (4 ng); lane 4, 60 TU (8 ng); lane 5, $120 \mathrm{TU}$ (16 ng); lane 6, $240 \mathrm{TU}(32 \mathrm{ng}) ;$ lane 7, amido black stain of blotted CIPE extract (240 TU and $12 \mu \mathrm{g}$ of total protein per lane). $B$, Neuronal number per $1 \times 5 \mathrm{~mm}$ strip width across each blot lane is shown with respect to the distance from the gel origin. The inset is a higher magnification photograph of the corresponding neuron-containing lanes shown in A. Molecular weight standards, run in adjacent lanes, were used to plot the standard curve.

versely, the procedure will allow the detection of apparent molecular weight changes (not accompanied by inactivation) after different treatments are applied to a purified CNTF before electrophoresis and blot analysis. Also, blots containing a CNTF could be exposed to experimental treatments after electrophoresis and blotting but before neuronal seeding to gain information on the stability of the bound CNTF activity.

The present technique might be adapted to the use of different neurons and to different neuronotrophic factors, thus extending the applicability to each of the above analyses. Trophic activities for different neurons have been observed in the same source material, and the modified approach would permit one to ascertain whether the activities rest with the same or with different polypeptides. Alternatively, the methodology might be adapted to the investigation of neurite-promoting or other neuron-modulating proteins, as well as to proteins affecting culture behaviors of glial or other probe cells.

Soluble versus bound CNTF. The present findings draw attention to an important question, namely, whether CNTF blotted to nitrocellulose expresses its activity while remaining bound or only upon release from its initially bound state. The rather strict localization of living neurons to the blotted CNTF excludes the possibility of a general release of the factor into the medium but does not entirely rule out the possibility of a limited local release of trophic molecules. This latter possibility, however, seems unlikely based on two observations (data not shown), namely, that (i) CNTF blots underwent no apparent loss of trophic competence even after having been stored for 3 weeks in PBS before the presentation of probe neurons, and (ii) treatments of CNTF blots with either $90 \%$ methanol or $8 \mathrm{M}$ urea overnight also failed to impose detectable reductions in trophic activity on the blot. This evidence favors (but does not prove) the view that, in this culture system, the probe neurons encounter the CNTF molecules in their bound state, rather than in the local humoral environment. The possibility of an immobilized trophic factor supporting neuronal survival in culture, also explored with NGF (e.g., 

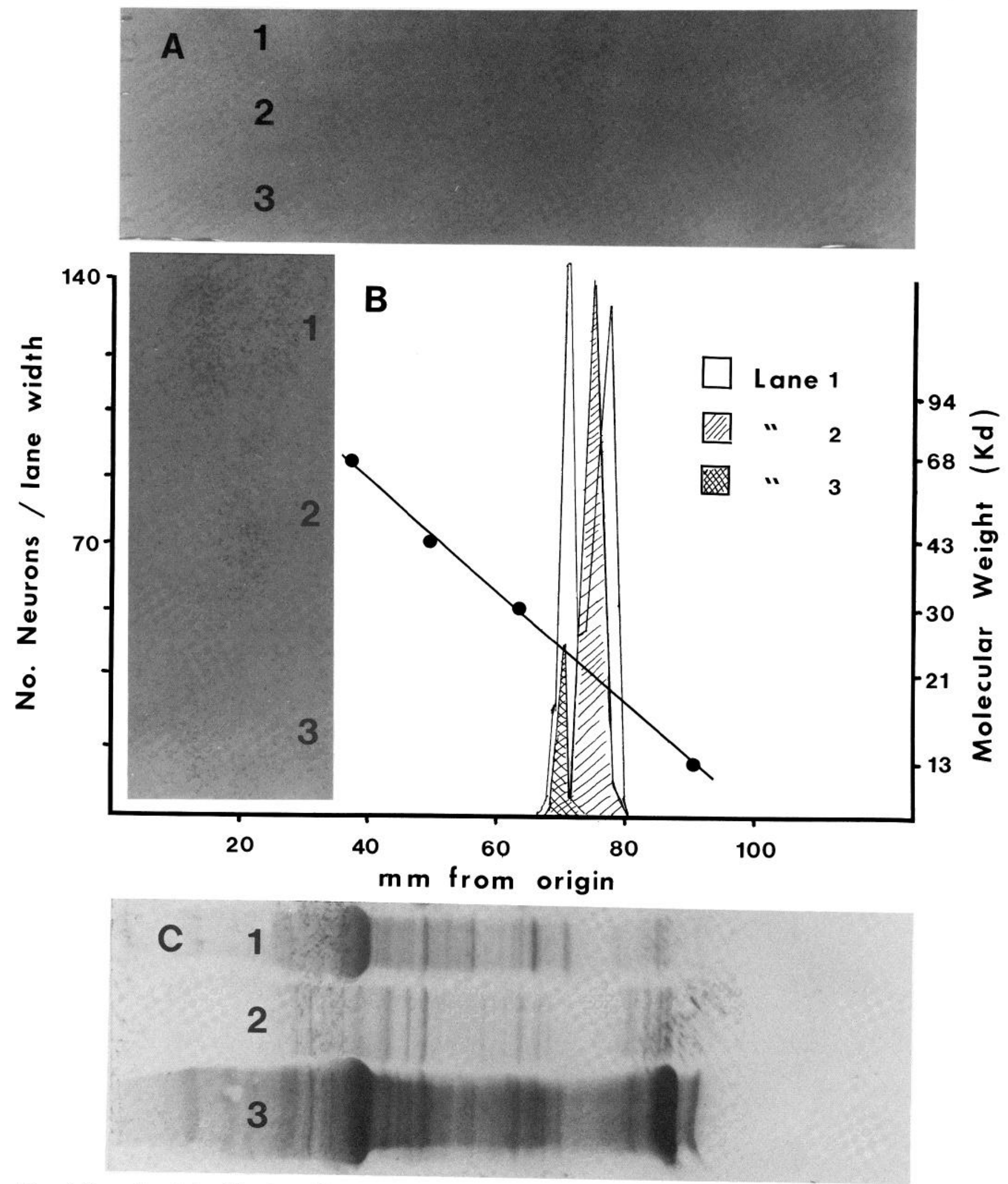

Figure 5. Recognition of nitrocellulose-bound CNTF within aqueous extracts of chick embryo eye, adult rat sciatic nerve, and Gelfoam sponges from adult rat brain lesions. Experimental conditions were as in Figure 4 except that ciliary neurons were cultured on nitrocellulose blots from SDS gels containing three adjacent sample lanes. A, Photograph of the fixed blot after culture showing neuronal survival on discrete areas: lane 1 , nerve extract (40 $\mu \mathrm{g}$ of protein and $7000 \mathrm{TU} / \mathrm{lane})$; lane 2, CIPE extract (20 $\mu \mathrm{g}$ and $400 \mathrm{TU})$; and lane 3, brain lesion fluid (200 $\mu \mathrm{g}$ and $100 \mathrm{TU})$. B, Neuronal numbers per $1 \times 5 \mathrm{~mm}$ strip across each blot lane are shown with respect to the distance from the gel origin. Inset in $B$, Larger magnification of the area in $A$ containing localized ciliary neuronal survival. Molecular weight standards, run in adjacent lanes, were used to plot the standard curve. $C$, Amido black stain of each extract containing the same amounts of protein indicated in $A$. 
Frazier et al., 1973; Oger et al., 1974), raises this interesting question of whether such a capability also exists in vivo.

\section{References}

Barbin, G., M. Manthorpe, and S. Varon (1984) Purification of the chick eye Ciliary Neuronotrophic Factor (CNTF). J. Neurochem. 43: 1468-1478.

Barde, Y. A., D. Edgar, and $H$. Thoenen (1982) Purification of a new neurotrophic factor from mammalian brain. EMBO J. 1: 549-553.

Bradshaw, R. A., and N. V. Costrini (1983) The structure and function of Nerve Growth Factor. In Growth and Maturation Factors, G. Guroff, ed., Vol. 1, pp. 1-30, John Wiley \& Sons, New York.

Davis, G. E., M. Manthorpe, and S. Varon (1985) Parameters of neuritic growth from ciliary ganglion neurons: Influence of laminin, Schwannoma polyornithine-binding neurite promoting factor and ciliary neuronotrophic factor. Dev. Brain Res. 17: 75-84.

Frazier, W. A., L. F. Boyd, and R. A. Bradshaw (1973) Interaction of Nerve Growth Factor with surface membranes: Biological competence of insolubilized Nerve Growth Factor Proc Natl. Acad. Sci. U. S. A. 70: 29312935.

Greene, L. A., and E. M. Shooter (1980) The Nerve Growth Factor: Biochemistry, synthesis and mechanism of action. Annu. Rev. Neurosci. 3: 353402.

Hayman, E. G., E. Engvall, E. A'Hearn, D. Barnes, M. Pierschbacher, and E. Ruostahti (1982) Cell attachirtenl un replicas of SDS polyacrylamide gels reveals two adhesive plasma proteins. J. Cell Biol. 95: 20-23.

Laemmli, U. K., and M. Favre (1970) Cleavage of structural proteins during the assembly of the head of bacteriophage T4. Nature 227:680-685.

Manthorpe, M., and S. Varon (1985) Regulation of neuronal survival and neuritic growth in the avian ciliary ganglion by trophic factors. In Growth and Maturation Factors, G. Guroff, ed., Vol. 3, pp. 77-117. John Wiley \& Sons, Inc., New York.

Manthorpe, M., S. D. Skaper, R. Adler, K. B. Landa, and S. Varon (1980) Cholinergic neuronotrophic factors: Fractionation properties of an extract from selected chick embryonic eye tissues. J. Neurochem. 34: 69-75.

Manthorpe, M., E. Engvall, E. Ruoslahti, F. M. Longo, G. E. Davis, and S. Varon (1983a) Laminin promotes neuritic regeneration from cultured peripheral and central neurons. J. Cell Biol. 97: 1882-1890.
Manthorpe, M., M. Nieto-Sampedro, S. D. Skaper, G. Barbin, F. M. Longo, t. R. Lewis, C. W. Cotman, and S. Varon (1983b) Neuronotrophic activity in brain wounds in the developing rat. Correlation with implant survival in the wound cavity. Brain Res. 267: 47-56.

Mosmann, T. (1983) Rapid colorimetric assay for cellular growth and survival: Application to proliferation and cytotoxicity assays. J. Immunol. Methods 65: $55-63$

Nieto-Sampedro, M., M. Manthorpe, G. Barbin, S. Varon, and C. W. Cotman (1983) Injury-induced neuronotrophic activity in adult rat brain: Correlation with survival of delayed implants in the wound cavity. J. Neurosci. 3: $2219-2229$.

Oger, J., B. G. W. Arnason, N. Pantazis, J. Lehrich, and M. Young (1974) Synthesis of Nerve Growth Factor by $L$ and $3 T 3$ cells in culture. Proc. Natl. Acad. Sci. U. S. A. 71: 1554-1558

Towbin, H., T. Staehelin, and J. Gordon (1979) Electrophoretic transfer of proteins from polyacrylamide gels to nitrocellulose sheets: Procedure and some applications. Proc. Natl. Acad. Sci. U. S. A. 76: 4350-4354

Varon, S., and R. Adler (1981) Trophic and specifying factors directed to neuronal cells. Adv. Cell. Neurobiol. 2: 115-163.

Varon, S., M. Manthorpe, and R. Adler (1979) Cholinergic neuronotrophic factors. I. Survival, neurite outgrowth and choline acotyltransfcrase activity in monolayer cultures from chick embryo ciliary ganglia. Brain Res. 173: $29-45$.

Varon, S., R. Adler, M. Manthorpe, and S. D. Skaper (1983a) Culture strategies for trophic and other factors directed to neurons. In Neuroscience Approached through Cell Culture, S. E. Pfeiffer, ed., Vol. 2, pp. 5377, CRC Press, Boca Raton, FL.

Varon, S., M. Manthorpe, F. M. Longo, nd L. R. Williams (1983b) Growth factors in regeneration of neural tissues. In Nerve, Organ, and Tissue Regeneration: Research Perspectives, F. J. Seil, ed., pp. 127-155, Academic Pres, Inc., New York

Varon, S., M. Manthorpe, and L. R. Williams (1984) Neuronotrophic and neurite promoting factors and their clinical potentials. Dev. Neurosci. 6 : 73-100.

Williams, L. R., M. Manthorpe, M. Nieto-Sampedro, C. W. Cotman, and S. Varon (1984) High ciliary neuronotrophic specific activity in rat peripheral nerve. Int. J. Dev. Neurobiol. 2: 177-180. 